\title{
Topographien der Vergänglichkeit
}

\author{
Vanitas, Alter und Gesellschaft bei Monika Maron und \\ Martin Walser
}

\begin{abstract}
In der deutschsprachigen Gegenwartsliteratur vollzieht sich die Auseinandersetzung mit dem Altern häufig unter Bezugnahme auf die tradierte VanitasMotivik. In den paradigmatischen Romanen, Monika Marons „Endmoränen“ und Martin Walsers „Angstblüte“, wird sie mit einer gesellschaftsdiagnostischen Raumsemantik verbunden, um auf die Prekarität derzeitiger gesellschaftlicher Alterswahrnehmung im Kontext sozioökonomischer Inferiorisierungen und (bio)medizinischer Unsterblichkeitsphantasmen hinzudeuten. Dies verändert insbesondere die ethischen Implikationen der Vergänglichkeitsreflexionen. Die Romane zeigen in Anlehnung an das Bildreservoir der spätmittelalterlichen, ars moriendi', wie ein christliches Ethos in der Gegenwart in ein säkulares transformiert wird, das darauf abzielt, ein gesamtgesellschaftliches Bewusstsein für die ,Natürlichkeit‘ des Alterns zu reetablieren.
\end{abstract}

\section{Vanitas, Alter und Gesellschaft: Literatursoziologische und raumtheoretische Perspektiven}

Als anthropologische Konstante konfrontiert das fortschreitende Altern scheinbar $\mathrm{zu}$ allen Zeiten und in allen Gesellschaften gleichermaßen mit Aspekten der Vergänglichkeit menschlichen Lebens. Die Entstehung, die Transformationen und die Konjunkturen des Vanitas-Topos als „spezifische[] Ausprägung eines historisch-anthropologischen Phänomens"1 verweisen wiederum auf die speziellen gesellschaftlichen und historischen Bedingungen seiner jeweiligen Thematisierung. ${ }^{2}$ Vergänglichkeitsreflexionen sind im Zuge ihrer Wiederkehr eingebunden in eine Bewegung aus gleichzeitiger Wiederholung und Differenz, die

1 Claudia Benthien und Victoria von Flemming. „Einleitung“. Paragrana. Internationale Zeitschrift für Historische Anthropologie 27.2 [Themenheft ,Vanitas. Reflexionen über Vergänglichkeit in Literatur, bildender Kunst und theoretischen Diskursen der Gegenwart‘. Hrsg. von dens.] (2018): 11-35, S. 11.

2 Seinen Ursprung hat der Vanitas-Topos bekanntlich im alttestamentlichen Buch Kohelet, im Übergang zur Frühen Neuzeit wird er schließlich neu gewichtet, rhetorisiert, ästhetisiert und

Ә Open Access. (c) 2021 Leonie Süwolto, publiziert von De Gruyter. (c) BY-NC-ND Dieses Werk ist lizensiert unter einer Creative Commons Namensnennung-Nicht-kommerziell-Keine Bearbeitung 4.0 International Lizenz. https://doi.org/10.1515/9783110716016-010 
sich mit Claudia Benthien und Victoria von Flemming als „Re-Semantisierung[]“3 der Vanitas-Denkfigur und ihrer Motive beschreiben lässt, deren Bedeutungsverschiebungen zuweilen gesellschaftsdiagnostisches Potential freisetzen. ${ }^{4}$

Diagnostiziert und archiviert die gegenwärtige Ubiquitarität des Motivkomplexes dementsprechend zuvörderst ein ausgeprägtes Vergänglichkeitsbewusstsein, das in der heutigen säkularen Gesellschaft prioritär die großen ,Reibungsverluste' zivilisatorischen Fortschritts wie z. B. die Klimakrise anprangert, ${ }^{5}$ verweist er dort, wo sich seine Motivik mit zeitgenössischen Altersdiskursen verbindet, unter anderem auf die soziokulturelle respektive ökonomische Prekarität des Alters in der Gegenwartsgesellschaft. Schließlich zeitigt die medizinische und biotechnologische Arbeit an der Unsterblichkeit eine zunehmende Pathologisierung des auf den ,natürlichen‘ Tod zustrebenden Alters, das im Zuge seiner potentiellen Vermeid-, Behandel- und Heilbarkeit stigmatisiert wird, ferner als nicht eben geringer ökonomischer Faktor den Generationenvertrag belastet und sozioökonomisch begründete Inferiorisierungen hervorbringt. ${ }^{6}$ Wo Modi der Unsterblichkeit wissenschaftlich plausibilisiert werden und damit in fast greifbare Nähe rücken, ${ }^{7}$ werden Alte, Siechende und Sterbende zu den verbleibenden inkorporierten Mahnmalen und Relikten eines Bedrohung und narzisstische Kränkung verheißenden memento mori. Sie erinnern an die Eitelkeit der menschlichen Exis-

semantisch angereichert. Vgl. Claudia Benthien. „Vanitas mundi. Der barocke VergänglichkeitsTopos in bildender Kunst, zeitbasierten Medien und Literatur der Gegenwart“. Frühe Neuzeit Späte Neuzeit. Phänomene der Wiederkehr in Literaturen und Künsten ab 1970. Hrsg. vom Nordverbund Germanistik. Bern u. a.: Lang, 2011. 87-108, S. 88. Aus literarhistorischer Perspektive lassen sich historische Konjunkturen des mit Vanitas verbundenen Motivkomplexes im Barock und im Fin de Siècle konstatieren.

3 Benthien und von Flemming 2018 (Anm. 1), S. 11.

4 Vgl. ebd., S. 11-12 und 17-19.

5 Der wachsende Bedeutungsverlust christlicher Leitbilder und eines christlichen Heilsversprechens, das Trost hinsichtlich der Begrenzung allen irdischen Lebens spendet, beeinflusst sicherlich die gegenwärtigen Thematisierungen von Vergänglichkeit stark.

6 Seit der Reform des Sozialversicherungssystems von 1957 werden die Renten- und Krankenversicherungen durch ein Umlageverfahren auf der Basis des Solidarprinzips finanziert. Spätestens seit den ersten Unkenrufen eines Altersstrukturwandels der Bevölkerung in den 1980er Jahren gerät dieses Prinzip zusehends unter Druck und es prägt sich in Zusammenhang mit der Finanzierbarkeit des Altersruhestands eine alarmistische Rhetorik aus, die zuweilen gar als apocalyptic demography bezeichnet wurde. Siehe dazu ausführlich: Leonie Süwolto. Altern in einer alterslosen Gesellschaft. Literarische und filmische Imaginationen. Paderborn: Fink, 2016, S. 87-93.

7 Zum (bio)medizinischen Altersdiskurs seit den 1980er Jahren vgl. ebd., S. 76-86. Zum wissenschaftlichen Streben nach ,Quasi-Unsterblichkeiten“ im Gegensatz zu theologischen Konzepten der Finalität siehe den Beitrag von Johannes Grössl in diesem Band. 
tenz angesichts einer biotechnologisch, medizinisch, kosmetisch und ökonomisch forcierten Jugendidolatrie. ${ }^{8}$

In der Literatur der Gegenwart gewinnt die beschriebene (soziale) Prekarität der Alterswahrnehmung häufig im Rahmen der Modellierung peripherer, heterotoper, hospitalisierter und gar kolonialisierter Räume Gestalt. ${ }^{9}$ Ihre Ästhetik rekurriert auf gesellschaftliche Ausgrenzungspraktiken, reflektiert sie zugleich kritisch und entwirft Handlungsräume im Potentialis, wie raumsoziologische Studien herausstellen, die seit dem Spatial Turn der 1980er und 1990er Jahre das methodisch-theoretische Spektrum der Literatur- und Kulturwissenschaften produktiv erweitern. ${ }^{10}$

Während die gegenwärtige literarische Reflexion des Alters sich also primär räumlicher Parameter zu bedienen scheint, operiert „der vanitas-Topos in der deutschen Barockliteratur [...] mit einem spezifischen Zeitkonzept. Sein zentrales Merkmal ist die Antizipation des Todes durch die sprachliche Herstellung einer (konzeptuellen) Simultanität von Gegenwart und Zukunft, ihrer unheimlichen Koexistenz." ${ }^{11}$ Paradigmatisch zeigt sich diese paradoxale, auf die (plötzliche) Präsenz des Todes im Leben verweisende Zeitlichkeit in der antithetischen Struktur von Andreas Gryphius’ Sonett „Es ist alles eitell“, das als Inbegriff der Vergänglichkeitsreflexion in der deutschen Barocklyrik gelten darf. Verse wie „Was dieser heute bawt/ reist jener morgen ein“ oder „Was itzund prächtig blüht sol bald zutretten werden“12 legen Zeugnis einer Gleichzeitigkeit des Ungleichzeitigen ab. Die temporale Synchronizität von ,heute‘ und ,morgen', ,itzund' und ,bald' manifestiert sich jedoch offensichtlich - wie die versifizierte Zerstörung des von Menschenhand gefertigten Bauwerks und der Vegetation zeigen - im Raum, so dass von einer chronotopischen Struktur des barocken

8 Dieser Begriff geht ursprünglich auf Jean Amérys Altersreflexion zurück. Vgl. Jean Améry. Über das Altern. Revolte und Resignation. Hrsg. von Monique Boussart. 5. Aufl. Stuttgart: KlettCotta, 1979.

9 Die Raummetaphorik der entsprechenden Romantitel ist in diesem Zusammenhang aufschlussreich, vgl. neben den hier behandelten Texten Annette Pehnts Das Haus der Schildkröten (2006) oder Arno Geigers Der alte König in seinem Exil (2011). Zum Zusammenhang von gegenwärtigem Altersdiskurs und literarischen Raumsemantiken siehe Süwolto 2016 (Anm. 6), S. 227-298.

10 Vgl. Jörg Dünne und Andreas Mahler. „Einleitung“. Handbuch Literatur \& Raum. Hrsg. von dens. Berlin: De Gruyter, 2015. 1-11.

11 Benthien 2011 (Anm. 2), S. 90.

12 Andreas Gryphius. „Es ist alles eitell“ [1637]. Gesamtausgabe der deutschsprachigen Werke 1: Sonette. Hrsg. von Marian Szyrocki und Hugh Powell. Tübingen: Niemeyer, 1963, S. 33-34. 
Vanitas-Topos auszugehen ist, ${ }^{13}$ wie auch bildkünstlerische Vergänglichkeitsreflexionen wie das Stillleben deutlich markieren. ${ }^{14}$

Meine an diesen Hintergrund anschließenden Ausführungen folgen daher der leitenden Überlegung, dass Filiationen des frühneuzeitlichen, insbesondere barocken Vanitas-Topos in der gegenwärtigen literarischen Auseinandersetzung mit dem Alter einerseits auf der Repetition des tradierten Motivinventars aufbauen, dieses andererseits variieren, indem seine chronotopische Struktur verbunden wird mit einer gesellschaftsdiagnostisch ausgerichteten (Alters-)Topographie.

Ferner ist $\mathrm{zu}$ beobachten, dass durch die Verbindung der tradierten VanitasMotivik mit einer auf die gegenwärtige gesellschaftliche Alterswahrnehmung verweisenden Raumsemantik auch ihre ethischen Implikationen modifiziert werden. Impliziert die Aufforderung zum memento mori im Kontext der christlichen Heilslehre noch die beständige Konfrontation des religiösen Selbst mit der eigenen Sterblichkeit zum Zweck einer gottgefälligen Lebensweise, scheint die ethische Herausforderung der Gegenwart primär darin zu bestehen, ein kollektives Bewusstsein für die Notwendigkeit und ,Natürlichkeit' des Sterbens in der gesellschaftlichen Mitte - um innerhalb der erkenntnisleitenden Raummetaphorik zu bleiben - zu reetablieren, um somit in zweiter Instanz den sozialen Status des Alters und der Alten in unserer Gegenwart zu problematisieren. Zu diesem Zweck kehren auch Elemente der spätmittelalterlichen Erbauungsliteratur, der ars moriendi, in der Gegenwartsliteratur unter veränderten Vorzeichen wieder und werden in Auseinandersetzung mit den gesellschaftlichen Verdrängungsmechanismen gegenüber Alter und Sterben ,re-semantisiert‘.

Im Folgenden sollen diese Überlegungen anhand von zwei thematisch besonders einschlägigen Romanen der deutschsprachigen Gegenwartsliteratur plausibilisiert werden: Monika Marons Endmoränen (2002) und Martin Walsers Angstblüte (2006) verdeutlichen schon durch ihre jeweiligen programmatischen Titelgebungen, dass sie nicht nur eine exponierte Vergänglichkeitsreflexion leisten, sondern

13 Der Begriff, der das Verhältnis von Raum und Zeit in einer Erzählung beschreibt, geht auf Michail Bachtin zurück. Vgl. Michail Bachtin. Chronotopos. Übers. von Michael Dewey. Frankfurt a. M.: Suhrkamp, 2008.

14 Die Sichtbarmachung der Gleichzeitigkeit des Ungleichzeitigen erfolgt im Vanitas-Stillleben über ein räumliches Nebeneinander von lebendigen und toten, bzw. von Fäulnis und Verderbnis befallenen Dingen im Raum, denn „verborgen inmitten der obszönen Schönheit und der opulenten Arrangements von exotischen Blumen und Früchten, [findet man] bereits einen kleinen braunen Fleck, ein halb verwelktes Blatt, oder irgendein anderes Indiz des bevorstehenden und doch schon gegenwärtigen - Verfalls. Zukunft und Gegenwart werden derart in einer einzigen bildlichen Darstellung verknüpft und synchronisiert.“ Benthien 2011 (Anm. 2), S. 92. Zur zeitgenössischen Adaption des Vanitas-Stilllebens siehe die Beiträge von Julia Catherine Berger und Verena Russlies in diesem Band. 
diese ausdrücklich in den Kontext von Naturphänomenen respektive natürlicher Prozessualität stellen: Die symbolträchtige Endmoräne als natürliche Landmarke des maximalen Vorstoßes des ewigen Eises eines Gletschers kodiert eine paradoxale Temporalität - als steiniger Ausläufer eines Gletschers impliziert sie Vergänglichkeit; als ewiges Eis verweist sie auf eine diesseitige Ewigkeit der Natur. Der ,Angstblüte، als letztem Blütentrieb einer Pflanze vor ihrem Sterben zum Zweck der Fortpflanzung inhäriert nicht nur eine widersprüchliche Zeitlichkeit, sie verweist unmittelbar auf die Ikonographie bildkünstlerischer Vanitas-Reflexionen im Barock, insbesondere im Blumenstillleben, ${ }^{15}$ und stellt sich ferner in die Tradition frühneuzeitlicher Liebeslyrik und ihrer Aufforderung zum carpe diem, insbesondere zum erotischen Lebens- und Liebesgenuss. Bei Walser kehrt diese als „schamlose Altherrenerotik ${ }^{\star 16}$ wieder, wie sie in ähnlich offensiver Weise z. B. Philip Roth entworfen hat. $^{17}$

\section{Wiederkehr und Differenz: Vanitas in Monika Marons Endmoränen}

Marons Roman reflektiert das Leiden der alternden Protagonistin Johanna an der empfundenen Sinnlosigkeit ihres Daseins vor dem Hintergrund zentraler Motive des tradierten Vanitas-Topos. Die Zweifel Johannas, vor allem gegenüber der Relevanz des eigenen Schaffens als ehemals systemkritische Autorin von Künstlerbiographien in der DDR, deren Widerständigkeit durch die deutschdeutsche Wiedervereinigung längst ihren Fluchtpunkt verloren hat, paaren sich im Roman mit einem durch das fortschreitende Altern ausgelösten Gefühl melancholischen Weltüberdrusses. ${ }^{18}$

15 Vgl. Benthien 2011 (Anm. 2), S. 92: „Wunderschöne und illusionistisch präzise gemalte Blumen stehen in voller Blüte, aber damit auch schon kurz vor dem Verwelken - in der Fülle und Pracht ist Vergänglichkeit schon inhärent, muss konstant mit reflektiert werden." Siehe auch: Norbert Schneider. Stilleben. Realität und Symbolik der Dinge. Köln: Taschen, 1989, S. 134-149. 16 Burkhard Müller. „Der neue Walser: ,Angstblüte‘. Obszöne Genialität“. Süddeutsche Zeitung (11.05.2010). https://www.sueddeutsche.de/kultur/der-neue-walser-angstbluete-obszoenegenialitaet-1.419713 (Zugriff am 22.12.2020).

17 Zur Vergänglichkeitsreflexion bei Roth vgl. Benthien 2011 (Anm. 2), S. 102-104.

18 Der Roman stellt sich somit in die Tradition der contemptus-mundi-Literatur: Die Geringschätzung alles Weltlichen wird bei Maron jedoch nicht durch ein christliches Ethos bedingt, sondern durch einen empfundenen Sinnverlust und daran anschließenden Rückzug, der sich eindeutig aus weltlichen Beweggründen speist. Die Protagonistin büßt ihren Platz in der Mitte der Gesellschaft ein, weil sie überzeugt ist, ihren Anforderungen nicht mehr gerecht zu werden. 
Die prekäre Selbstwahrnehmung der weiblichen Hauptfigur speist sich dabei maßgeblich und unverkennbar aus der gesamtgesellschaftlichen Wahrnehmung des Alters als „katastrophaler Kostenfaktor für die Krankenkassen“"19. Der pejorativen Haltung der Gesellschaft entspricht schließlich die periphere Raumsemantik der Erzählung. Die durch die Topographie der Landschaft bedingte Isolation der fiktiven ostdeutschen Provinz Basekow und ihrer alternden Bewohnerinnen entlarvt diese regelrecht als ,Alterskolonie“ im Sinne einer Betrachtung der Raumanordnung aus einer an den Postcolonial Studies geschulten Perspektive. ${ }^{20}$ Die Identifikation kolonialer Strukturen in der Gestaltung des Handlungsortes rechtfertigt sich dabei nicht nur durch eine dem Roman subkutan eingeschriebene kritische Auseinandersetzung mit dem deutsch-deutschen Verhältnis, die von einer westlichen Kulturhegemonie ausgeht. Darüber hinaus verweist die Einöde auf eine „symbolische Apartheid“ des Alters in der Gegenwartsgesellschaft im Allgemeinen, die - wie Rüdiger Kunow gezeigt hat - Praxen der Kolonialisierung insofern vergleichbar sei, als sie Strukturen „entmachteter Sozialität“ visualisiere. ${ }^{21}$

Die apostrophierte Abgeschiedenheit des Ortes von der pulsierenden Kulturlandschaft Berlins und die Unwegsamkeit der Verbindung zwischen Stadt und Land, die den Zugang zur Ortsgemeinschaft nahezu verunmöglicht, setzen in raumsoziologischer Perspektive eindrucksvoll die gesellschaftlichen Verdrängungsleistungen gegenüber Alter, Krankheit und Tod ins Licht. Zudem manifestiert sich in der Modellierung der Handlungsräume ein metaphorisches Kräftemessen zwischen Natur und Kultur. Die Raumgestaltung des Romans drängt dieses Deutungsangebot nahezu auf, insbesondere da die Stadt als Schauplatz wesentlicher Kulturleistungen unmittelbar mit Unsterblichkeitsphantasien der Stadtbewohner*innen in Verbindung gebracht wird. Johannas Ehemann, der Literaturwissenschaftler und ausgewiesene Kleist-Forscher Achim, weigert sich z. B. zunehmend, die Stadt für eine Stippvisite aufs Land zu verlassen, um im urbanen Raum unermüdlich „de[m] Dienst an [Kleists] Unsterblichkeit“ zu frönen und damit indirekt auch an seiner eigenen Unsterblichkeit. ${ }^{22}$ Der Ort Basekow dagegen ist im Gegensatz zur Stadt

19 Monika Maron. Endmoränen. Frankfurt a. M.: Fischer, 2002, S. 55-56.

20 Der Ausdruck „Bewohnerinnen“ wurde bewusst gewählt, denn es handelt sich tatsächlich mehrheitlich um ortsansässige Frauen, die auf die Geschlechtsspezifik des Altersdiskurses, seine strukturelle Verwandtschaft mit Weiblichkeitsdiskursen schließen lassen. Vgl. dazu Süwolto 2016 (Anm. 6), bes. S. 53-56.

21 Rüdiger Kunow. „,Ins Graue‘. Zur kulturellen Konstruktion von Altern und Alter“. Alter und Geschlecht. Repräsentationen, Geschichten und Theorien des Alter(n)s. Hrsg. von Heike Hartung. Bielefeld: Transcript, 2005. 21-43, S. 37.

22 Explizit thematisiert wird das Unsterblichkeitsphantasma Achims in der Romanfortsetzung: Monika Maron. Ach Glück. Frankfurt a. M.: Fischer, 2006, S. 70-71. 
übriggeblieben, zurückgelassen von der Zeit wie die als sanfte Hügel sich breitenden Endmoränen, die ihn umschlossen. [...] [E]in Sechsunddreißigseelenort, in den ein ausgefahrener, nach Regen nur noch an den Rändern passierbarer Sandweg hineinführte und eine im Acker verendende Traktorspur wieder hinaus. ${ }^{23}$

Der morastige Sand und Ackerboden der Landschaft drohen, die Passant*innen aus der Stadt beinahe spurlos in sich aufzunehmen, wie auch die auf den Ackerbau als primäre Kulturleistung verweisende Traktorspur unversehens im lehmigen Boden Basekows verendet, um erneut zum Teil einer als übermächtig gekennzeichneten, sterblichen Natur zu werden. Die eröffnete Dichotomie des Raumes verbürgt zunächst das gesellschaftliche Bestreben, das Alter als Reminiszenz der Vergänglichkeit menschlichen Lebens in einen durch die Endmoränen markierten Bannkreis zu stellen. Doch zeigt sich schließlich im Romanverlauf, dass die Verbindung von Vergänglichkeit, Alter und Natur in der Raumsemantik vor allem einem ethischen Impetus folgt. Die sukzessive Inversion der zivilisierten und domestizierten Flora zur übermächtigen Natur impliziert die Notwendigkeit einer Renaturalisierung des Alters im öffentlichen Bewusstsein, das dem kulturoptimistischen Glauben an seine potentielle Überwindbarkeit entgegentritt, um der anhaltenden gesamtgesellschaftlichen Ausgrenzung von Alter, Krankheit und Sterben Einhalt zu gebieten.

Bezüglich der Reflexion von Vergänglichkeit und Alter macht Maron schließlich Gebrauch von verschiedenen Filiationen (vor-)barocker Vanitas-Motivik, die sich vor der ländlichen Kulisse entfalten. Das auftretende alternde, zuweilen von Krankheit gezeichnete Figurenpersonal erinnert etwa an die seit dem Spätmittelalter gebräuchlichen skulpturalen Manifestationen des memento mori in Gestalt von elfenbeinernen Wendekopf-Plastiken, die ursprünglich als Elemente des Rosenkranzes dem ${ }^{\star}$ der gläubigen Christ ${ }^{\star}$ in die eigene Sterblichkeit versichern und in der Gebetspraxis die Bitte um Beistand in der Todesstunde haptisch untermauern sollten. ${ }^{24}$ Ihre modernen Inkarnationen bevölkern zahlreich die Naturlandschaft Basekows. Die entworfenen Frauenkörper sind wie die Wendekopf-Plastiken doppelgesichtig, als wandelnde Vexierbilder repräsentieren sie die paradoxe Zeitlichkeit der Vanitas. In ihrer „wächserne[n] Haut“ scheine der Tod hervor, „wie in einem Röntgenbild““ ${ }^{25}$ Die Gegenwart des Todes im alten, jedoch noch vitalen Körper zeichnet der Protagonistin Johanna gar „die sichtbare Gravur der Greisenhaftig-

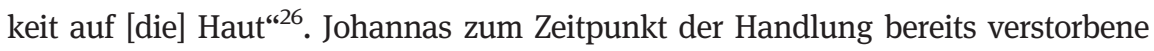

23 Maron 2002 (Anm. 19), S. 20-21.

24 Vgl. Andrea von Hülsen-Esch und Hiltrud Westermann-Angerhausen (Hrsg.). Zum Sterben schön. Alter, Totentanz und Sterbekunst von 1500 bis heute. Regensburg: Schnell + Steiner, 2006, S. 77.

25 Maron 2002 (Anm. 19), S. 11.

26 Ebd., S. 26. 
Freundin Irene, deren Lebensende mit der Landschaft Basekows assoziativ verknüpft ist, da ihr Tod zeitlich mit dem Erwerb des Landsitzes der Familie zusammenfiel, erscheint in ihrer Physiognomie gar als idealtypische Inkorporation des topischen Tödleins, jener kleinen, rundplastischen Figur in der Tradition der barocken memento-mori-Kunst, deren nur partiell skelettierte Erscheinung sie als Leichnam ausweist, während sie gleichzeitig Tätigkeiten der Lebenden ausführt. ${ }^{27}$ Die Beschreibung der siechenden Irene rekurriert unzweifelhaft auf jenes vertraute Bildinventar: Während Gliedmaße und Antlitz noch teilweise mit Haut und Muskeln überspannt sind, ist die Evidenz des Todes in Gestalt des durchscheinenden menschlichen Skeletts und des Totenschädels kaum noch zu leugnen:

Ihre Beine standen stockdünn auf langen Füßen in orthopädischen Schuhen, die mageren, behaarten Arme reichten fast bis zu den Knien, weil Irenes Wirbelsäule zwischen den Schulterblättern verkrümmt war und ihren Oberkörper um zehn oder zwölf Zentimeter verkürzte. [...] [S]kelettdünn, kahlköpfig, die großen Zähne zwischen den erschlafften Lippen, die halbrund aus den Höhlen quellenden Augäpfel mit mattfarbener Iris. ${ }^{28}$

Die Darstellung menschlicher, vor allem weiblicher Körper im Roman, deren Extremitäten Fleisch gewordene Gleichzeitigkeiten des Ungleichzeitigen von Leben und Tod repräsentieren, wird erzählerisch enggeführt mit der Raumgestaltung. Die Physiognomien der alternden Frauen werden in den umgebenden Raum externalisiert und so buchstäblich zum Teil einer von Werden und Vergehen bestimmten Naturlandschaft. In der Figurenzeichnung der verwitweten Bäuerin Friedel Wolgast kulminiert die Transgression von Körper und Raum:

Bis zum Tod ihres Mannes hatte Friedel Wolgasts Körper Jahr für Jahr eine Schicht zugelegt, wie Jahresringe um einen Baum, und sich mit der Zeit zu einem in dieser Gegend üblichen derben, konturlosen Frauenkörper ausgewachsen, mit schweren Brüsten und der gleich darunter ansetzenden Wölbung des Bauches, von ähnlicher Masse an der Hinterseite im Gleichgewicht gehalten. Schon während der Krankheit ihres Mannes war Schicht um Schicht von Friedel Wolgasts Matronenleib abgeschmolzen, der nun dabei war, seine letzte Gestalt im irdischen Leben anzunehmen. ${ }^{29}$

Die ausgeprägte Naturmetaphorik der Körpertransformation impliziert nicht nur eine Renaturalisierung des Lebenslaufs und damit des Alters. Sie reflektiert auch die sozioökonomische Prekarität des Alters, insofern die beschriebene ,Schneeschmelze' des Leibes, seine Metamorphose im doppelten Verweis erstens auf die ,natürliche‘ Prozessualität von Altern und Sterben rekurriert, zweitens die prekäre Versorgungssituation der alternden Witwe kodiert.

27 Vgl. Von Hülsen-Esch und Westermann-Angerhausen 2006 (Anm. 24), S. 27.

28 Maron 2002 (Anm. 19), S. 7 und 14.

29 Ebd., S. 60. 
Vermittelt über den Zusammenhang von Körper und Raum gewinnt schließlich ein ethisches Anliegen zunehmend an Kontur, das den fortschrittsoptimistischen Glauben an eine finale Domestikation der Natur, in diesem Fall des Alters und Sterbens, mit der Transgression des Kräfteverhältnisses von Kultur und Natur beantwortet, das in der Raumgestaltung sichtbar wird. Die Topographie Basekows erfährt eine sukzessive Umdeutung: Manifestieren sich in ihr zunächst die gesellschaftlichen Ausgrenzungspraktiken, steht sie im Verlauf der Erzählung zunehmend ein für eine Emphase der in ihr zur Sichtbarkeit gelangenden natürlichen Prozessualität.

Ebenso wie die Vergänglichkeit der natürlichen Vegetation Friedel Wolgasts Körpertransformation in „seine letzte Gestalt im irdischen Leben“30 metaphorisierte, erzeugt die Naturlandschaft Basekows auch für die verzagte Protagonistin einen Reflexionsraum der eigenen Vergänglichkeit. Die Sterblichkeit der Natur wird jedoch zunächst noch durch die Aussicht auf die Beständigkeit der kulturellen Hinterlassenschaften des Menschen konterkariert, die durch das Landhaus der Protagonistin repräsentiert werden, dessen Dachfirst anagrammatisch die Frist menschlicher Lebenszeit zu markieren scheint:

Angesichts der entlaubten, vor der weiten erdfarbenen Landschaft kaum sichtbaren Bäumchen, rechnete ich aus, wann sie den Dachfirst unseres Hauses erreicht haben würden, und mußte an meinen Tod denken, der, gemessen an der gewünschten Baumhöhe, plötzlich sehr nahe schien. ${ }^{31}$

Wenig später entscheidet der Romanverlauf jedoch final über die Dominanzverhältnisse, indem das räumliche Nebeneinander von Wachstum des Baumes und Höhe des Hauses in der Beschreibung eines von der Natur zerstörten Kulturguts synchronisiert wird.

\footnotetext{
In jedem Frühjahr hatten wir das neuerliche Zerstörungswerk des Winters registriert. Am Ende wuchsen drei Birken aus seinem löcherigen Dach. Durch die Reste von mürbem Putz leuchtete das Mauerwerk wie rohes Fleisch, die Fensterhöhle, die Fensterhölzer faulten und brachen, der bröselige Stein der Eingangstreppe verwandelte sich nach und nach wieder in den Sand. Wie ein geschundener Körper starb das Schloss langsam vor unser aller Augen. ${ }^{32}$
}

Die Deskription der von der natürlichen Vegetation durchstoßenen Schlossruine lässt einen deutlichen intertextuellen Bezug zur Vanitas-Reflexion in der Barocklyrik erkennen. Wie Gryphius’ eingangs zitiertes Sonett „Es ist alles eitell“

30 Ebd.

31 Ebd., S. 25.

32 Ebd., S. 143-144. 
gestaltet die Textpassage den Übergang von einem lebendig pulsierenden zu einem skelettierten Körper und schließlich zur Asche als Gleichzeitigkeit: „Was itzt so pocht vndt trotzt ist morgen asch und bein“33. Die Gleichzeitigkeit von Leben und Tod, die Gryphius primär durch die in der Antithetik seiner Verse enthaltenen Temporaladverbien erzeugt, Maron wiederum durch das in der Raumgestaltung modellierte Nebeneinander von Bestand und Verfall, weicht im Zitat schlussendlich dem Eindruck der sukzessiven zeitlichen Entwicklung eines ,Nach und Nach“. Das Skandalon des Todes schwindet zusehends im Maße einer durch die Vegetation präfigurierten natürlichen Prozessualität von Altern und Sterben.

Die Transformation der anthropomorphisierten Schlossfassade in den Sandboden des Provinzortes repräsentiert in nuce die Bedeutungsverschiebungen, welche die tradierte Vanitas-Motivik im gesamten Roman erfährt: Ein überliefertes Motivinventar kehrt vornehmlich in der Figurengestaltung und der Modellierung alter Körper wieder, um schließlich über Externalisierungen und Transgressionen von Körper und Raum mit einer Raumsemantik verbunden zu werden, welche in raumsoziologischer Perspektive erstens auf die Prekarität gegenwärtiger Alterswahrnehmung rekurriert. Zweitens formuliert es jedoch prospektiv einen Handlungsimperativ hinsichtlich des gesamtgesellschaftlichen Umgangs mit dem auf die Eitelkeit des menschlichen Lebens verweisenden Alter. Die erzählerische Verbindung einer exponierten Vergänglichkeitsreflexion mit der Gestaltung einer den Naturgesetzen unterworfenen Landschaft stellt sich als Versuch der Renaturalisierung des Alters im Bewusstsein einer Gesellschaft dar, deren Fortschrittsoptimismus die Alten als Reminiszenz des Faktums der Sterblichkeit buchstäblich und metaphorisch an ihre Ränder und Peripherien verdrängt hatte. Doch während die aufgerufenen Traditionslinien der Vanitas-Reflexion durch ihre christlichen Kontexte irdische Vanitas mit dem Versprechen auf eine himmlische aeternitas kompensieren, ${ }^{34}$ spendet im Roman Endmoränen der Glaube an die Einbindung des Menschen in einen göttlichen Heilsplan längst keinen Trost mehr.

33 Gryphius 1963 (Anm. 12), S. 33.

34 Gemäß Ferdinand van Ingen ist die Vergänglichkeitsreflexion der Barocklyrik „von einem überkommenen christlichen Todesverständnis geprägt“. Mit Einzelanalysen exemplarischer Gedichte etwa von Gryphius oder Hoffmannswaldau weist er nach, dass die lyrischen Auseinandersetzungen mit Vanitas auf einem himmlischen Ewigkeitsversprechen aufbauen. Vgl. Ferdinand van Ingen. Vanitas und Memento Mori in der deutschen Barocklyrik. Groningen: Wolters, 1966, S. 11. 


\section{Vom locus terribilis zum locus amoenus: Vergänglichkeitsreflexionen in Martin Walsers Angstblüte}

Die Suche nach Trost angesichts des nahenden Todes ist eines der zentralen Themen von Martin Walsers Roman Angstblüte (2006). Im Zentrum der Handlung steht der 70-jährige Investmentberater Karl von Kahn, der fest an die lebenserhaltende Kraft der Geldvermehrung glaubt. Angesichts der durch sein Alter erlittenen Kränkungen und zunehmenden wirtschaftlichen Misserfolgs begibt er sich dennoch auf die Suche nach einem letzten amourösen Abenteuer und verliebt sich Hals über Kopf in die junge und schöne Schauspielerin Joni Jetter. Ähnlich wie Marons Protagonistin Johanna wähnt sich auch Walsers männliche Hauptfigur als Opfer einer durch die hegemonialen Altersdiskurse der Zeit bedingten Marginalisierung:

In Wirklichkeit gibt es unser Alter nicht. Es ist eine Mache der Alarmisten. Von meinem und deinem Alter wissen sie nichts. Für die Alarmisten sind wir Statistikfutter. Sie reden von uns, wie der Farbenblinde von der Farbe redet. Über mein Alter und dein Alter gibt es keine Auskunft. Die produzierten Horizonte aus nichts als Gefahren, um sich als Retter aufspielen zu können. ${ }^{35}$

Auch hier manifestieren sich die skizzierten Abwehrmechanismen der Gegenwartsgesellschaft gegenüber Alter und Sterben in der Modellierung eines abseitigen Raumes, der als karger Gebirgszug unverkennbare Züge eines locus terribilis außerhalb der menschlichen Gemeinschaft trägt: ${ }^{36}$ „Sie schreiben über unser Alter wie über ein Gebirge, das sie nur vom Flugzeug aus kennen. Vom Drüberhinfliegen. Sie wissen nicht, wie das ist, in diesem Gebirge zu leben.“37 Die beklagte Unkenntnis der räumlichen Gegebenheiten der Naturlandschaft lässt auf die umfassenden Verdrängungsmechanismen schließen, denen sich der Alternde in der Gesellschaft ausgesetzt sieht.

Noch wähnt sich der Investor jedoch in relativer Sicherheit, insofern er glaubt, durch spekulative Geldvermehrung den nahenden physischen Tod überwinden zu können. Durch seine „Poesie des Zinseszins“38

35 Martin Walser. Angstblüte. Reinbek bei Hamburg: Rowohlt, 2006, S. 25.

36 Zum locus terribilis vgl. Klaus Garber. Der locus amoenus und der locus terribilis. Bild und Funktion der Natur in der deutschen Schäfer- und Landlebendichtung des 17. Jahrhunderts. Köln: Böhlau, 1974, S. 240-263.

37 Walser 2006 (Anm. 35), S. 25.

38 Manuel Bauer. „Die unwirkliche Poesie des Zinseszins: Fiktionalität der Geldwirtschaft in Martin Walsers Roman ,Angstblüte““. Finanzen und Fiktionen. Grenzgänge zwischen Literatur und Wirtschaft. Hrsg. von Christine Künzel und Dirk Hempel. Frankfurt a. M.: Campus, 2011. 201-216. 
mehrung durch die „Wieder- und Wieder- und Wiederanlage des Erworbenen“39 begeistert von Kahn bevorzugt hochbetagte Anleger`innen für langfristige Investitionen, vor allem im Bereich medizinischer Technologien. Seine Geldgeschäfte florieren buchstäblich, ebenso wie ökonomische Erwägungen mit einer auf Vanitas verweisenden Naturmetaphorik durchwebt sind. Die wirtschaftlichen Erträge, die rhetorisch mit der Blütenpracht der umgebenden Flora und Fauna überblendet werden, implizieren dergestalt immer schon ihre eigene Vergänglichkeit. Es ist die Rede vom „Werte-Gewächshaus“40 , vom „Markt als Naturgeschehen“41 und schließlich wird die explizite gedankliche Auseinandersetzung mit der eigenen Sterblichkeit begleitet von einem symbolträchtigen Vergleich von Baumund Zinswachstum:

Er sollte sich allmählich eine Grabstelle suchen. [...] Aber jedesmal, wenn er über diese Mauer schaute, dachte er, daß er hier nicht beerdigt sein möchte. Die Grabsteine standen zu eng nebeneinander. Überfüllt wie die U-Bahn, dieser Friedhof. Bis er in die Osterwaldstraße einbog, ging er immer, als sei er in Eile. Dann aber ließ er sich protegieren von den Bäumen. Die Bäume gaben alles, was man an sie hindachte, reichlich zurück. Zinsen, dachte er und ging noch langsamer. ${ }^{42}$

Während der erste Romanteil von Optimismus und dem festen, pseudoreligiösen Glauben an eine ökonomisch zu erzielende Immortalität gekennzeichnet ist wenngleich das Faktum der Vergänglichkeit dieser Perspektive durch die Naturmetaphorik sichtbar eingeschrieben ist -, kommt es infolge der fatalen Begegnung mit der Schauspielerin Joni Jetter $\mathrm{zu}$ einer lust- und genussvollen Antizipation der eigenen Endlichkeit an einem symbolträchtigen locus amoenus, der sich als Gegenentwurf zu dem die Altersängste der Gesellschaft repräsentierenden locus terribilis erweist.

Wie bereits Maron ihre Altersreflexion in den Kontext der Vanitas-Lyrik des Barock stellte, rekurriert auch Walser auf tradierte Motive der Barock-, insbesondere der Liebeslyrik des Christian Hoffmann von Hoffmannswaldau. Während bereits die Titelgebung des Romans Angstblüte auf die (letzte) Möglichkeit sexuellen Genusses im Angesicht der Vergänglichkeit in der Manier barocker Liebeslyrik anhebt (so formuliert in Versen wie „Ich werde mich betrüben/ | So diese rose stirbt | Und ohne lust verdirbt“"43), wird auch die Beschreibung von

39 Walser 2006 (Anm. 35), S. 24.

40 Ebd., S. 23.

41 Ebd., S. 24.

42 Ebd., S. 103.

43 Christian Hoffmann von Hoffmannswaldau. „[WAs willtu Doris machen / Brich deinen stoltzen Geist]“. Benjamin Neukirchs Anthologie. Herrn von Hoffmannswaldau und andrer Deut- 
Jonis physischer Schönheit durch die intertextuelle Bezugnahme auf die im Barock besungene „Vergänglichkeit der Schönheit“44 doppelbödig: Zwar steht die junge Schöne - um in der Metaphorik des Romans zu bleiben - in voller Blüte, doch birgt ihre alles überstrahlende Erscheinung erste Anzeichen des Verfalls. Ihr Zahnstatus verweist auf den Beginn körperlicher Degeneration; und dies umso mehr als dieser unmittelbar mit der Morbidität der behandelnden Zahnärzte Jonis verbunden wird: „Jetzt sei ihr doch wieder der Zahnarzt gestorben. Der vierte Zahnarzt stirbt ihr einfach weg. Autounfall, Herzinfarkt, Gehirntumor, Leberzirrhose. Sie traut sich nicht mehr, zu einem Zahnarzt zu gehen. Das ist für den doch das Todesurteil.“ ${ }^{45}$

Darüber hinaus rekurriert die äußerliche Beschreibung der Aktrice auf ein relativ stereotypes Bildinventar der vergänglichkeitsbewussten Liebeslyrik des Barock. Während des ersten Aufeinandertreffens von Karl und Joni preist der Anlageberater das ,[h]erausragende Objekt des Schönheitsdiskurses [...] im barocken vanitas-Gedicht und der entsprechenden bildenden Kunst, das Partialobjekt der weiblichen Brüste“46. Es heißt: „Die Hauptsache wurde offen, halboffen ausgestellt. Die Brüste. ${ }^{\text {47 }}$ Am deutlichsten offenbart sich der intertextuelle Re-

schen auserlesener und bißher ungedruckter Gedichte 1 [1697]. Hrsg. von Angelo George de Capua und Ernst Alfred Philippson. Tübingen: Niemeyer, 1961, S. 385-387.

44 In Anlehnung an den Titel eines der bekanntesten Sonette von Christian Hoffmann von Hoffmannswaldau: „Vergänglichkeit der Schönheit“. Benjamin Neukirchs Anthologie. Herm von Hoffmannswaldau und andrer Deutschen auserlesener und bißher ungedruckter Gedichte 1 [1697]. Hrsg. von Angelo George de Capua und Ernst Alfred Philippson. Tübingen: Niemeyer, 1961, S. 46-47. 45 Walser 2006 (Anm. 35), S. 252.

46 Benthien 2011 (Anm. 2), S. 104. Zur Fetischisierung von Körperteilen im Barock vgl. Hartmut Böhme. „Erotische Anatomie. Körperfragmentierung als ästhetisches Verfahren in Renaissance und Barock“. Körperteile. Eine kulturelle Anatomie. Hrsg. von Claudia Benthien und Christoph Wulf. Hamburg: Rowohlt, 2001. 228-253, bes. S. 232-236. Für den Lobgesang auf die weiblichen Brüste gibt es allein in der Lyrik Hoffmannswaldaus unzählige Beispiele. In einem der bekanntesten Sonette, „Vergänglichkeit der Schönheit“, heißt es: „Es wird der bleiche Todt mit seiner kalten Hand | Dir endlich mit der Zeit um deiner Brüste streichen." Hoffmannswaldau 1961 (Anm. 44). In „[ALbanie / gebrauche deiner Zeit]“ preist das lyrische Ich die weibliche Brust mit den Worten „Die äpfel/ so auff deinen brüsten prangen/ / Sind unsre lust/ und süsse anmuthssee“ (Christian Hoffmann von Hoffmannswaldau. „[ALbanie /gebrauche deiner zeit]“. Benjamin Neukirchs Anthologie. Herrn von Hoffmannswaldau und andrer Deutschen auserlesener und bißher ungedruckter Gedichte 1 [1697]. Hrsg. von Angelo George de Capua und Ernst Alfred Philippson. Tübingen: Niemeyer, 1961, S. 70-71), im Sonett „Beschreibung vollkommener Schönheit“: „Zwo brüste/ wo rubin durch alabaster bricht“. (Christian Hoffmann von Hoffmannswaldau. „Beschreibung vollkommener Schönheit“. Benjamin Neukirchs Anthologie. Herm von Hoffmannswaldau und andrer Deutschen auserlesener und bißher ungedruckter Gedichte 1 [1697]. Hrsg. von Angelo George de Capua und Ernst Alfred Philippson. Tübingen: Niemeyer, 1961, S. 87-88).

47 Walser 2006 (Anm. 35), S. 202. 
kurs auf die barocke Liebeslyrik jedoch in der Deskription von Jonis Gesicht während des Geschlechtsverkehrs. Karl beobachtet den lustvoll verzehrten Mund der Geliebten:

Ihr ungeschminkter Mund war eine Landschaft. Eine Flußlandschaft. Zwei Ufer, die zueinander wollten. [...] Die Lippen waren immer voller geworden. Der Mund bebte und schwankte wie ein Schiff bei immer höherem Wellengang und zerriß, verlor alle Form, war nur noch eine Verzerrung. ${ }^{48}$

Unschwer kann Jonis als tobende See metaphorisierter Mund als imaginäres Homologon des weiblichen Genitals identifiziert werden, das den Seefahrer in seinen Untiefen buchstäblich versenkt. ${ }^{49}$ Im Sonett „Florida“ macht Hoffmannswaldau von einer vergleichbaren Metaphorik Gebrauch, um den Liebesgenuss zu besingen, der das lyrische Ich sogleich mit der eigenen Sterblichkeit konfrontiert:

Mein Schiff treibt lufft und wind / mich treibet lieb und brunst

Ich muß in Florida den steiffen ancker sencken/ [...]

Da will ich / wann und wie es das verhängnüß heist

Mich in die grosse zahl der todten lassen setzen. ${ }^{50}$

Liebe und Verhängnis, rauschhafte Vereinigung und Tod werden hier wie dort in der Überblendung von Körper- und Landschaftstopographie synchronisiert, insofern auch Walsers Imagination des sexuellen Verschmelzens in Gestalt der einander zustrebenden Flussufer auf mythologische Vorstellungen des Totenreichs rekurriert. ${ }^{51}$ Im Zusammensein mit Joni versenkt sich Karl buchstäblich und sinnbildlich in die Naturlandschaft, deren Semantik einst unverkennbar die gesellschaftlichen Altersängste repräsentierte. Durch die Verbindung von Körper und Landschaft im Geschlechtsakt - „Das rabiate Genießen des Verblühtseins einer Frau. Die Gemeinsamkeit des Zerfalls als die endgültige Ge-

48 Ebd., S. 249-250.

49 Nicht nur die physiognomisch begründbare Ähnlichkeit erlaubt es, Jonis Mund als Analogon der Vagina zu lesen. Auch, dass ihre Lippen hier als Gradmesser sexueller Lust fungieren, legt diesen Schluss nahe. Insbesondere die Psychoanalyse hat auf die Homologie der Gesichtsund Unterleibsanatomie aufmerksam gemacht, gemäß Freud steht sie im Kontext einer „Verschiebung von unten nach oben [...] im Dienste der Sexualverdrängung“. Sigmund Freud. Die Traumdeutung. 7. Aufl. Frankfurt a. M.: S. Fischer, 1987, S. 392.

50 Christian Hoffmann von Hoffmannswaldau. „Florida“. Benjamin Neukirchs Anthologie. Herm von Hoffmannswaldau und andrer Deutschen auserlesener und bißher ungedruckter Gedichte 2 [1697]. Hrsg. von Angelo George de Capua und Ernst Alfred Philippson. Tübingen: Niemeyer, 1965, S. 10.

51 Angespielt wird hier sicherlich auf die Bedeutung der Styx in der griechischen Mythologie. Joni kann im Sinne dieses Analogieschlusses als Inkarnation von Charon identifiziert werden. 
meinsamkeit.“52 - wird letztere analog zur Vanitas-Motivik in der Barocklyrik sakralisiert. Sie wird zum gelobten Land der rauschhaften Konfrontation mit der eigenen Endlichkeit und einer (göttlichen) Ewigkeit, die bei Walser jedoch nicht mehr einer christlichen Vorstellung entspricht, sondern verschiedene christliche, mythische sowie mystische Vorstellungen und Kulte in sich vereint. Dem Schoß der Frau wird gehuldigt als Begegnungsstätte von Vanitas und aeternitas. Bei Walser rekurriert bereits die Namensgebung der weiblichen Protagonistin Joni in ihrer engen lautlichen Verwandtschaft zum tantrischen Begriff ,Yoni‘ auf das weibliche Genital, das durch seine stilisierte runde Darstellungsform in vielen Kulturen als Unendlichkeitssymbol bzw. als Symbol (göttlicher) schöpferischer Kraft fungiert. ${ }^{53}$ Im Barockgedicht, etwa in „ALbanie/ gebrauche deiner zeit“ von Hoffmannswaldau, fungiert der weibliche Schoß dagegen noch eindeutig als Tor zu einem christlich konnotierten Paradies: „Albanie / soll denn dein warmer schooß / So öd und wüst / und unbebauet liegen? | Im paradieß da gieng man nackt und bloß / | Und durffte frey die liebes-äcker pflügen“. 54

Wo Walsers Protagonist sich zunächst in Unsterblichkeitsphantasmen verstieg, um die Anzeichen des an die ,natürliche“ Sterblichkeit gemahnenden Alterungsprozesses und seiner gesellschaftlichen Inferiorisierung umfassend $\mathrm{zu}$ verdrängen, zeitigen die Rekurse auf Vanitas-Reflexionen in der barocken Liebeslyrik über die Verbindung von weiblichem Körper und vergänglicher Naturlandschaft letztlich eine euphorische Antizipation des Sterbenmüssens. Diese baut jedoch nicht mehr - wie noch im Barock - auf einem christlichen Glaubensbekenntnis auf, sondern auf einer Unendlichkeitsvision, die Züge monotheistischer respektive christlicher Religionen trägt, jedoch auch Elemente antiker Mythologie und fernöstlicher Religionen und Kulte einschließt.

52 Walser 2006 (Anm. 35), S. 469. Die Überblendung von weiblichem Körper und der umgebenden Flora gehört zur Topik des barocken Liebesgedichts in der Tradition von Hoffmannswaldau. Besonders deutlich wird diese etwa im Sonett „Beschreibung vollkommener Schönheit“, wo es heißt: „Ein mund/ der rosen führt und perlen in sich heget / | [...] / Zwey wangen/ wo die pracht der Flora sich beweget“. Hoffmannswaldau 1961 (Anm. 46), S. 88.

53 Vgl. Peter und Anneliese Keilhauer. Die Bildsprache des Hinduismus. Die indische Götterwelt und ihre Symbolik. Köln: Du Mont, 1986, S. 167-169. Die Rundungen der weiblichen Körperform werden von Walser unmittelbar mit der Namensgebung seiner weiblichen Romanfiguren verbunden, z. B.: „Gundi nackt. Gundula Powolny. So mußte sie heißen, der Name war wie ein Körperteil. Rundweichfließend. Gundipowolny.“ Walser 2006 (Anm. 35), S. 44.

54 Hoffmannswaldau 1961 (Anm. 46), S. 71. 


\section{Neue Perspektiven auf Alter und Sterben: Ethik und Ästhetik der Raumgestaltung bei Maron und Walser als zeitgenössische ars moriendi?}

Die Gewissheit, dass ,Leben lernen und sterben können“ eng verwandt sind -

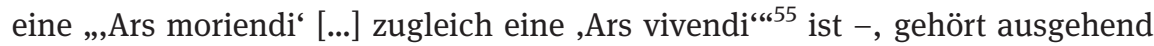
von der theologischen Literatur des Mittelalters sicherlich längst zu den Gemeinplätzen der Auseinandersetzung mit Vergänglichkeit. Damit betont werden soll die ethische Bedeutung von Vergänglichkeitsreflexionen, denn ein gutes und sinnvolles Leben ist offenkundig abhängig von der Akzeptanz seiner zeitlichen Begrenzung, jedoch in der christlichen Tradition ebenso konstitutiv angewiesen auf den Trost eines posthumen Ewigkeitsversprechens.

Die vormoderne ars moriendi hielt in diesem Sinne das Bewusstsein der Sterblichkeit und Endlichkeit irdischen Daseins im Leben des religiösen Selbst stets präsent und gab ihm als Sterbekunst gleichzeitig Anweisung für ein gutes, d.h. gottgefälliges Leben. Denn die Rettung der Seele des`der Gläubigen im Leben wurde zur konstitutiven Bedingung der Überwindung der Furcht vor dem Sterben des Körpers und dementsprechend eines angstfreien Sterbens in Erwartung der Aufnahme der Seele in die göttliche Ewigkeit:

Dort diente der Hinweis auf die Vergänglichkeit des Lebens und der Dinge dazu, den Menschen zu mahnen, daß auch er ein vergängliches und kurzlebiges Geschöpf ist. Der Inhalt der Warnung ist wohl dieser, daß der Mensch sein flüchtiges Dasein im Lichte der Ewigkeit betrachten soll: Rechne immer mit dem Tod und lebe christlich. ${ }^{56}$

Als Teil spätmittelalterlicher Erbauungsliteratur hatten die überlieferten artes moriendi einen fest verankerten Sitz im Alltagsleben des ${ }^{\star}$ der gläubigen Christin, zum Teil waren sie in sehr kleinformatigen Ausgaben „zur ständigen Mitnahme und zum praktischen Gebrauch vorgesehen “57.

55 Van Ingen 1966 (Anm. 34), S. 5.

56 Ebd., S. 89.

57 Von Hülsen-Esch und Westermann-Angerhausen 2006 (Anm. 24), S. 11. Bereits auf der Ebene der Materialität der Editionen lässt sich ein auf die Verbindung von Marons Roman Endmoränen zu den spätmittelalterlichen artes moriendi bezogenes Gedankenexperiment wagen: Die Pocket-Format-Edition der im S. Fischer-Verlag erschienenen Romane, die auf der Homepage unter dem Reiter ,Taschenbibliothek' rubriziert sind, ist aussagekräftig in Bezug auf den alltagspraktischen Gebrauchswert der Texte, entspricht ihre handliche Größe doch einem zur ständigen Mitnahme geeigneten Taschenkalender. Wesentlich bedeutsamer sind über diesen sehr pragmatischen Zugriff hinaus jedoch die inhaltlichen Korrespondenzen zwischen Marons Roman und der spätmittelalterlichen Erbauungsliteratur. 
Wie jedoch eine säkulare ars moriendi ohne das von ihr in Aussicht gestellte, Trost spendende göttliche Heilsversprechen aussehen kann, scheint im Kontext gegenwärtiger medialer und künstlerischer Auseinandersetzungen mit dem Sterben die Frage der Stunde zu sein. ${ }^{58}$ Dies, insofern sich nicht nur die Frage individueller Sinnstiftung angesichts des Faktums des Todes stellt. Sondern auch, da im Sinne des literatursoziologischen Zuschnitts meiner Ausführungen angesichts gegenwärtiger gesamtgesellschaftlicher Verdrängungsleistungen gegenüber Alter und Sterben auch die Frage nach einem adäquaten kollektiven Umgang mit der Vergänglichkeit menschlichen Lebens zu beantworten ist, der den ,Alten“ unserer Gesellschaft ein gutes Leben ermöglicht. Marons und Walsers Romane gehen der Frage nach einem guten Leben in der letzten Lebensphase angesichts der Bedrohung durch den nahenden Tod und in Auseinandersetzung mit gesellschaftlichen Denunziationen gleichermaßen nach. Beide greifen dabei auf symbolträchtige Raumkonstellationen illustrierter artes moriendi zurück, so dass in der Raumsemantik der Erzähltexte eine frühneuzeitliche bzw. spätmittelalterliche Vanitas-Ästhetik und eine säkulare, auf die Wahrnehmung von Alter und Sterben in der Gegenwartsgesellschaft bezogene Ethik des memento mori zusammengeführt werden.

Die bebilderten Darstellungen der artes moriendi machen in Bezug auf die zu betrachtende Verbindung von Ethik und Ästhetik der Raumgestaltung in den Romanen von einer topisch zu nennenden Verräumlichung der zeitlichen Differenz von Vanitas und aeternitas, Vergänglichkeit und Ewigkeit, Gebrauch, insofern „Aufbau und Inhalt [...] in allen Büchern der Bilder-Ars [...] nahezu gleich“59 sind. Sie inszenieren die Sterblichkeit des Körpers und die Unsterblichkeit der Seele des`der Gläubigen als gattungstypische Begegnung zwischen demder Siechenden, den umherstehenden Abschiednehmenden, den verführenden Dämonen und der auf die göttliche Ewigkeit verweisenden Dreifaltigkeit im Raum. Das zeitliche Ineinandergreifen von Vanitas und aeternitas wird in der bildkünstlerischen Gestaltung des Sterbebettes als Transitraum gestaltet. Trost spendet demder Sterbenden sowie den Angehörigen die im Raum bereits sicht- und greifbare Repräsentanz der göttlichen Ewigkeit (Abb. 1).

58 Ein Artikel in der Wochenzeitung Die Zeit zum Thema ,Die neue Sichtbarkeit des Todes“ etwa vermutet, in den unendlichen Weiten des virtuellen Raumes des World Wide Web und seinen sich niemals erschöpfenden Speicherkapazitäten den säkularen „Himmel auf Erden, eine [neue] Civitas Dei“ identifiziert zu haben. Thomas Assheuer. „Die neue Sichtbarkeit des Todes“ Die Zeit 48 (19.11.2009). https://www.zeit.de/2009/48/Sichtbarkeit-des-Todes (Zugriff am 22.12.2020). Es handelt sich hier um eine Besprechung des Bandes von Thomas Macho und Kristin Marek (Hrsg.). Die neue Sichtbarkeit des Todes. Paderborn: Fink, 2007.

59 Von Hülsen-Esch und Westermann-Angerer 2006 (Anm. 24), S. 12. 


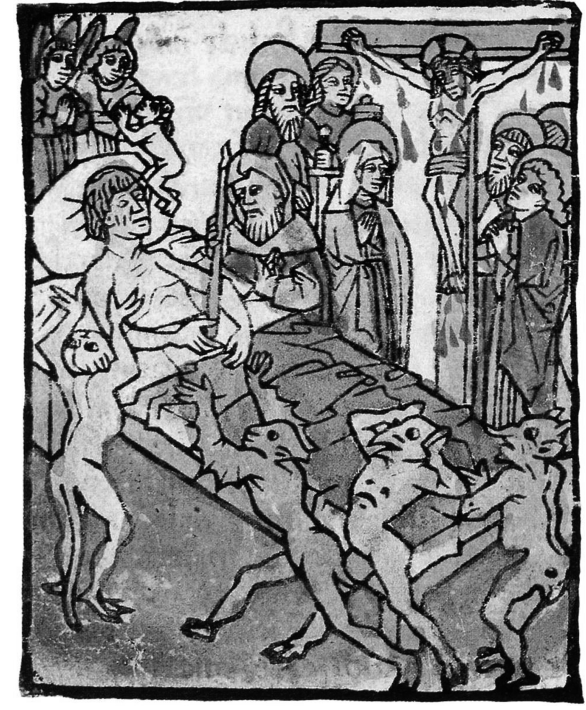

Abb. 1: unbekannter Künstler: Die Stunde des Todes, Holzschnitt aus Blockbuch, um 1460.

Obschon Marons Roman nicht illustriert ist, stellt sich der Rekurs auf die Tradition der artes moriendi vor allem über seine exponierte Sprachbildlichkeit her. Dergestalt lässt die Romanerzählung eine der Ikonographie der ,Bilder-Ars ${ }^{6}$ vergleichbare Szenerie vor den Augen der Leserschaft entstehen:

Nur nachts, wenn ich nicht schlafen konnte, weil die Fliederäste gegen die Dachrinne schlugen oder der Marder im Dach tobte und ich im oberen Stockwerk des Hauses saß wie auf einem Schiff im dunklen Meer, abgeschnitten von den anderen Menschen und allem, was hätte Halt bieten können, [...] [ließen mich] die ächzenden Bäume und der stoßweise atmende Wind [...] fürchten [...], daß ich ihresgleichen war. ${ }^{60}$

Das Gefühl der Drangsal durch die dämonisch anmutenden Naturgewalten weicht schließlich unversehens ihrer euphorischen Antizipation als Vorboten einer über die Eitelkeit des menschlichen Lebens hinausgehenden Ewigkeit der Natur, die den sterbenden Menschen in sich aufnimmt:

Selbst als ich in Basekow den Gewittern zusah oder dem Sturm, der in gewaltigen Wellen das Korn peitschte, empfand ich vor allem eine Genugtuung, weil die Macht keine Menschenmacht war, weil sie keinem Gesetz gehorchte und keiner Regierung, weil sie die Garantie war für einen größeren, der Lächerlichkeit unseres eigenen Lebens entzogenen

60 Maron 2002 (Anm. 19), S. 49. 
Zusammenhang. Der Gedanke, eine Kreatur dieser undurchschaubaren, endlosen Welt zu sein, stattete mich gegenüber der Tatsache, daß ich den idiotischen Gesetzen einer ebenso idiotischen Menschenmacht unterlag, mit unbestreitbaren Rechten aus. ${ }^{61}$

Obwohl es sich nicht tatsächlich um das Sterbebett der Protagonistin handelt, erinnert das evozierte Bild der furchterfüllten, im Bett liegenden Johanna an die illustrierten Sterbeszenen der artes moriendi, schließlich wird sie hier von einer Ahnung der Endlosigkeit und Ewigkeit erfüllt, an der sie durch die Anerkenntnis ihrer natürlichen Kreatürlichkeit partizipiert. Auch hier wird die Gleichzeitigkeit von Vanitas und aeternitas in der Raumgestaltung wahrnehmbar, nur werden die Vorboten der göttlichen Ewigkeit in den christlichen artes bei Maron durch die Anwesenheit der anthropomorphisierten, zunächst dämonisch erscheinenden Naturgewalten im Raum ersetzt. Wie die Aussicht auf die Unsterblichkeit der Seele des Gläubigen in der vormodernen Sterbekunst spendet die Renaturalisierung des alternden Körpers im Roman Trost, verheißt sie doch die Garantie für einen größeren, der Lächerlichkeit des eigenen Lebens entzogenen Zusammenhang. ${ }^{62}$ Und auch in Marons Roman birgt die hoffnungsfrohe Aussicht auf ein gutes Sterben gleichzeitig die Aussicht auf ein gutes, zuversichtliches Leben durch die Akzeptanz seiner zeitlichen Begrenzung: Die Anerkenntnis des Faktums der Sterblichkeit eines buchstäblich renaturalisierten Körpers stellt allererst die Weichen für die 2006 erschienene Fortsetzung der Dilogie Ach Glück, in der die Zeichen auf Aufbruch stehen und die Protagonistin deutlich gestärkt aus der ländlichen Einöde emporkommt. ${ }^{63}$

Während die Modellierung der einsamen Gebirgslandschaft im Kontext von Walsers gesellschaftskritischer Altersreflexion eindeutige Merkmale des locus terribilis trägt, wie er in der Literatur des 17. Jahrhunderts als „wilde[r] Ort abseits von den von Menschen bewohnten oder aufgesuchten Stätten“64, idealtypisch als Wüste, Wald, Gebirge oder Insel konzipiert wird, stellt sich eine später im Romanverlauf entworfene Naturimago als idealisierter Ort der Entgrenzung

61 Ebd., S. 24.

62 Die ekphrastische Schilderung im Roman synchronisiert und kondensiert die von den artes moriendi idealtypisch illustrierten Szenen der Anfechtungen, der Bestärkung und Mahnung und des Trostes in einer einzigen. Vgl. von Hülsen-Esch und Westermann-Angerer 2006 (Anm. 24), S. 11-12.

63 Der Roman Endmoränen ist Teil einer Dilogie. In der Fortsetzung Ach Glück bricht die gestärkte Protagonistin Johanna zu einer Reise nach Mittelamerika auf, um sich auf die Suche nach der Avantgarde-Künstlerin und Literatin Leonora Carrington zu begeben. Vgl. Maron 2006 (Anm. 22). Zur Bedeutung dieser Reise im Kontext der prekären gesellschaftlichen Wahrnehmung des Alters vgl. Süwolto 2016 (Anm. 6), S. 285-295.

64 Garber 1974 (Anm. 36), S. 242. 
von Endlich- und Ewigkeit dar. Dieser kann unschwer in der Tradition des locus amoenus als Lustort und Ort der Liebenden verortet werden: ${ }^{65}$

Ein unendlich schöner oder paradiesisch schöner Traum also. Von Anfang an. Eine von allem, was stören könnte, befreite Traumwelt. Mehr Menschen, als man auf einen Blick fassen kann. Alle beschäftigt mit Zärtlichsein. Keine Farbe, die an eine andere Farbe stößt. Nur Übergänge. Auch die Bäume, die Sträucher, die Häuser, die Brücken, die Flüsse, die Blumen, alles geht in alles über. Auch das, was man hört. Eine Musik der reinen Vollkommenheit. Hervorgebracht von keinem Instrument. Alles findet einem Mädchen zuliebe statt. Ist sie aus Tau oder Samt oder Licht? Sie ist aber auch ein Mädchen, das geht und steht uns sich bückt und streckt und sogar Sprünge macht. Bei den Sprüngen ist sie länger in der Luft, als man das für möglich hält. Man staunt. Ihre grenzenlose Wesens- und Körperschmiegsamkeit, ihre vollkommene Zutunlichkeit bewirkt nichts Geschlechtliches. [...] Sie kommt zu ihm. Er sitzt auf einem von blühender Kapuzinerkresse überwucherten Thron. Sie setzt sich auf ihn. Sie kriegt ihn zu spüren. Dann das unaufhaltsame Hinausgleiten aus ihr. ${ }^{66}$

Obschon die amöne Naturbeschreibung augenscheinlich an die Ikonographie christlicher Paradiesvorstellungen angelehnt ist, rekurriert Walser in der Schilderung des Idylls keinesfalls auf ein christliches Heilsversprechen, auf das der betagte Karl hoffen darf. Die in den illustrierten christlichen artes moriendi präsente Zusammenführung von Vanitas und aeternitas am bzw. im Sterbebett ist jedoch auch im Roman Angstblüte im paradiesischen Idyll strukturell angelegt: Der Todgeweihte sitzt auf einem das Sterbebett ersetzenden Thron, die anwesenden Menschen substituieren die Trauergemeinde, das Mädchen, dessen transzendente Erscheinung als Lichtgestalt die christliche Bildtradition der Aureole aufgreift, steht ein für die in den artes moriendi in Aussicht gestellte Entgrenzung von Endlich- und Ewigkeit. Seine Nacktheit rekurriert jedoch zugleich auf die Darstellungstradition der mythologischen Nymphen als Personifikationen der Naturkräfte. In dieser doppelten Verweisstruktur ist der Bedeutungswandel des von Walser imaginierten Ewigkeitsversprechens angedeutet: Eine von Werden und Vergehen gekennzeichnete Naturlandschaft wird sakralisiert, jedoch ist sie keine terra domini mehr. Im Sterben, das hier mitunter recht profan als kopulativer respektive reproduktiver Akt dargestellt ist, wird der Mensch Natur, wird er eingebunden in bzw. rückgebunden an eine natürliche Prozessualität. Präfiguriert wird diese Renaturalisierung des menschlichen Lebenslaufs durch das Bild der den Thron überwuchernden Kriechpflanze, das wiederum nicht nur die Einswerdung des sterblichen Menschen mit der Natur illustriert, sondern ferner die vollzogene Umkodierung der im himmlischen Thron symbolisierten göttlichen Macht zu einer

65 Vgl. ebd., S. 173-199.

66 Walser 2006 (Anm. 35), S. 475. 
säkularen Apotheose der Natur. Das Moment der lustvollen Hingabe des Sterbenden an die Naturkräfte auf der Ebene der Handlung korrespondiert mit einem formalästhetisch sichtbaren Wendepunkt in der Erzählung, der sich abermals an die Konstruktion des vergänglichen Körpers in der Barocklyrik anlehnt. Nicht nur auf der Handlungsebene des Romans antizipiert Walser analog zum barocken Liebesgedicht das Moment des Eindringens in den weiblichen Schoß als Tor zum (säkularen) Jenseits, auch die Poetik des Erzähltextes stellt eine Verbindung zu den vergänglichkeitsbewussten Sonetten des Barock her. Ebenso wie Hartmut Böhme eine Erotisierung und Fetischisierung in der Barocklyrik Hoffmannswaldaus primär in der Konstruktion eines „ornamental-rhetorischen“ Textkörpers identifiziert, ${ }^{67}$ lässt sich das Erscheinungsbild des Textkörpers bei Walser analytisch fruchtbar machen: Im Moment der lustvollen Hingabe, der zugleich das Moment der finalen Selbstaufgabe und des männlichen Kontrollverlusts repräsentiert, zerbirst buchstäblich der durch den Blocksatz in Form gezwungene Textkörper in einen scheinbar unkontrollierten, wie die Kriechpflanze vermeintlich wild wuchernden Flattersatz.

Die anfängliche Schilderung eines öden, felsigen und isolierten Gebirges als symbolträchtiger locus terribilis der gesellschaftlichen Ausgrenzung von Alter und Vergänglichkeit weicht im Romanverlauf schließlich einer euphorischen, gar rauschhaften Einswerdung mit der Natur am locus amoenus, durch die Alter und Sterben zunächst renaturalisiert und ferner aus dem gesellschaftlichen Abseits des locus terribilis zurück ins Bewusstsein einer imaginierten Gemeinschaft der Sterblichen („Mehr Menschen, als man auf einen Blick fassen kann““68) gebracht werden.

In der Modellierung von Marons Endmoränenlandschaft und Walsers amönem Idyll werden Ästhetik und Ethik des Vanitas-Topos unter den Bedingungen des gegenwärtigen gesellschaftlichen Umgangs mit Alter, Sterben und Tod reflektiert. Bekannte Elemente des Vanitas-Motivkomplexes, insbesondere aus dem Kontext der Barockliteratur, werden erzählerisch eingeflochten in die skizzierten Einöden, die wiederum symbolisch einstehen für den gesamtgesellschaftlichen Umgang mit den sichtbaren Zeichen der Vergänglichkeit in einer Zeit biotechnologischen, medizinischen und kosmetischen Anti-Agings. Gleichzeitig stehen die entworfenen Topographien ein für eine ihrer Semantik eingeschriebene Renaturalisierung des alternden und sterbenden Körpers, d. h. für eine Überwindung eines fortschrittsoptimistischen Glaubens an die Eliminierung des Todes

67 Böhme 2001 (Anm. 46), S. 241-242.

68 Walser 2006 (Anm. 35), S. 475. 
im Zuge zunehmender Kulturalisierung. Dergestalt vertreten sie auch ein ethisches Anliegen: Sie fordern auf zum memento mori, zur Bewusstmachung und Vergegenwärtigung der menschlichen Sterblichkeit, um in Anlehnung an die spätmittelalterliche ars moriendi und ihr Bildinventar die Teilhabe an einer säkularen Ewigkeit der Natur in Aussicht zu stellen.

\section{Abbildungsnachweis}

Abb. 1 @ gemeinfrei 